\title{
Automatic Detection of Road Signs to Control Vehicle Speed
}

\author{
Anuja Nanal \\ Dept. of Computer \\ Engineering \\ Pimpri Chinchwad \\ College of Engg \\ SPPU, Pune, India
}

\author{
Pooja Motwani \\ Dept. of Computer \\ Engineering \\ Pimpri Chinchwad \\ College of Engg. \\ SPPU, Pune, India
}

\author{
Pragati Pawar \\ Dept. of Computer \\ Engineering \\ Pimpri Chinchwad \\ College of Engg. \\ SPPU, Pune, India
}

\author{
Rajat Nirhale \\ Dept. of Computer \\ Engineering \\ Pimpri Chinchwad \\ College of Engg. \\ SPPU, Pune, India
}

\author{
Rahul Patil \\ Dept. of Computer \\ Engineering \\ Pimpri Chinchwad \\ College of Engg. \\ SPPU, Pune, India
}

\begin{abstract}
Nowadays many accidents are occurring because of the to negligence of road-signs while most driving of the vehicles. There are various zones specified for an area where the rules must be followed. The advancement in the processor technology and microcontrollers has come up with a new style so as to avoid such accidents in seeing traffic sign boards along the road. So to tell the driver about the safety zones and to automatically maintain the speed is accomplished by use of RF technology. The main objective of this project is to devise an Electronic Display controller meant for controlling vehicle speed and monitors the zones, and which can also display the speed to the rf reader with the help of unit attached in the car. This system can be adopted to abate the accidents caused due to speeding. This paper presents a new way to control the speed of the vehicles at remote places for particular time. The project has two parts: Zone status transmitter unit and Electronic Display and Control unit. The road-sign signal is received from the RFtransmitter on the sign boards in the zone, the vehicle's Electronic Display Controller Unit informs the driver to reduce the speed according to the zone, further it waits for driver to reduce speed and in case of no response by the driver, reduces the speed of vehicle automatically.
\end{abstract}

\section{General terms}

Road safety, Speed control mechanism,speed detection.

\section{Keywords}

Automobile, RF, embedded system.

\section{INTRODUCTION}

It is evident that road accidents are increasing every year with a hefty amount. Recent studies show that so many fatal accidents are caused due to excessive speeds in places of sharp and acute turnings and junctions are present, also the change in the roadway like the road-work signs or unexpected obstacles. Railway-crossings, reverse bends and steep ascents and descents are the few of the road anomalies that an average drive may face while driving. Such road entities are showed by road signs. Compulsory road-signs enforce traffic laws; Cautionary roadsigns are installed in hazard prone areas to avoid accidents. Informative road-signs provide directions and guidelines, locations and other information that is useful to the drivers in that locality. However, oftenly most of the drivers miss the road signs. It is extremely difficult to simultaneously manage both the vehicles driving control and also keenly look for any road signs on the roads, to help in these situations various driver assistant systems are being developed. One of all is Cruise control system (CC) which is capable enough of maintaining the defined original speed and its later evolving version Adaptive Cruise
Control (ACC) which also allows to ensure the speed and keeps the automobile at pre-defined safer distance from the preceding vehicle. These systems

developed cannot detect the signs in various circumstances like when the speed is more or the roads are curvy or the roads are inclined to specific angle to oppose the physical forces. Later Curve Warning Systems (CWS) were designed to efficiently detect the curves in the roads by the use of Global Positioning System (GPS) and the digital maps accessed from the Geographical Information Systems (GIS) to warn driver about the curvy road which would be arriving. But these maps which is supposed to guide the vehicles about the curves need to be updated on a regular basis and are not useful if there are unpredictable road diversions or accidents. Here therefore we propose a dynamic model for speed control where system controls the vehicle according to the data frame signal that is transmitted by the RF transmitter attached to the road signs. The data frame is received by the microcontroller and then it will control the vehicle. This RFID-Based Intelligent vehicle speed controller system in which the passive RF transceivers are arranged on the road close to the position of real traffic signals. This is the model can also be further applied to improve the fuel efficiency in vehicles by maintaining the speed.

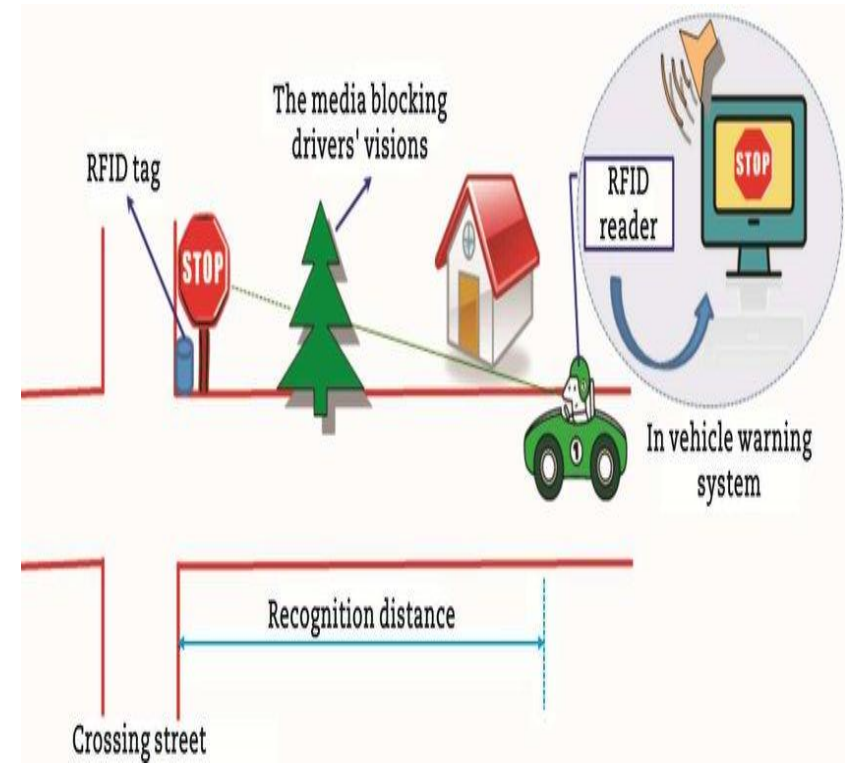

Figure 1.1 Traffic Signal posts equipped with RF Transmitter (left) Automobile equipped with the RF receiver (right) 


\section{LITERATURE SURVEY}

In literature survey, various kinds of speed control mechanisms or techniques have been proposed. Let us talk about the speed cotrol mechanism using RFID.

\subsection{Block Diagram:}

\subsubsection{Transmitter RF Tag:}

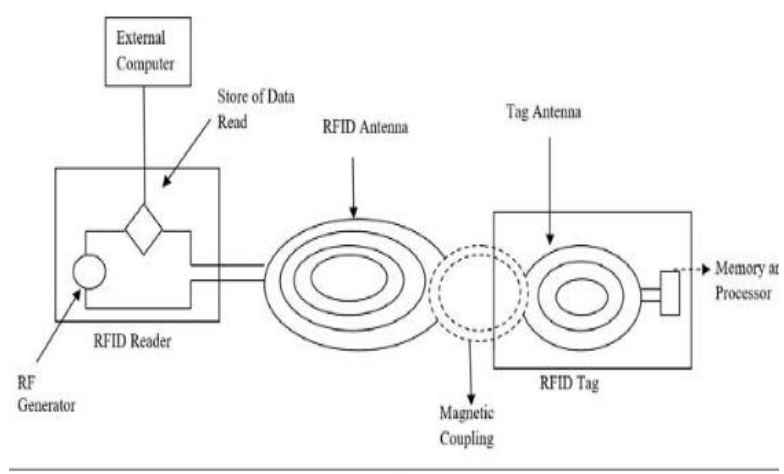

Figure 2.1: Passive RF Tag detection.

\subsubsection{Automobile Receiver Unit equipped with Display:}

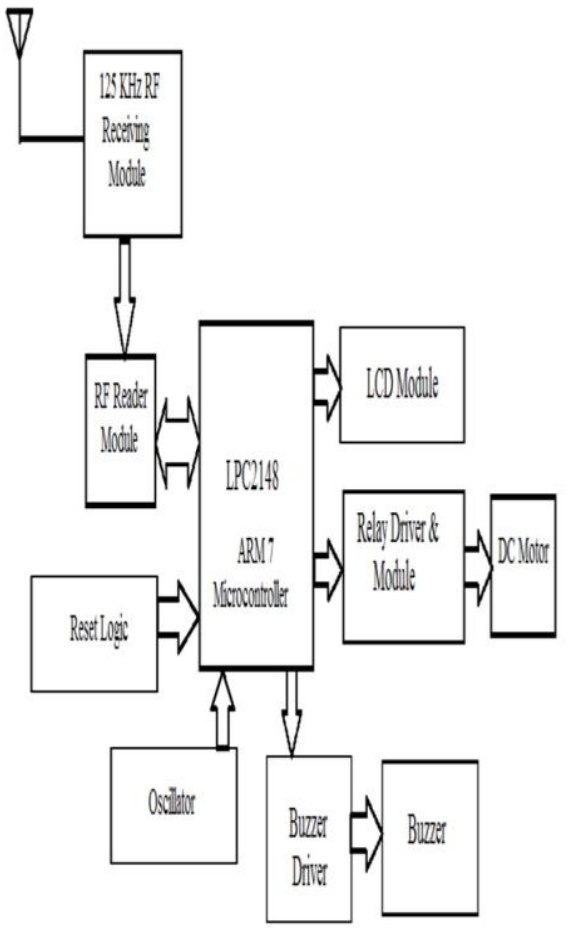

Fig 2.2: $\quad$ Electronic Controller and Display unit embedded in

\section{SYSTEM MODEL}

The system model for the Electronic Controller and Display Unit consists of following Hardware Modules:

\subsection{Microcontroller Module:}

The Arduino UNO is a widely used open-source micro controller board based on the Microchip at mega328 micro controller.The board is equipped with sets of digital and analog input/output (I/O) pins that are interfaced with the various expansion in the boards and some other circuits. The board has in all 14 Digital pins and 6 Analog pins. It is programmable with the Arduino IDE (Integrated Development Environment) via a type B USB cableLPC 2148 microcontroller is based on ARM7TDMI-S CPU with real-time emulation and embedded trace support. It couples microcontrollers with the embedded high speed flash memory and wide memory interface. Due to their low power consumption and tiny size, LPC 2148 are ideal for applications where miniaturization is a requirement, such as access control. It consists of 2 ports for interfacing with various devices and has RAM $(40 \mathrm{~kb})$ and Flash memory $(512 \mathrm{~kb})$ and also high speed performance at a speed of $60 \mathrm{MHz}$. The user is able to control module with an external device such as microcontroller or PC.

\subsection{RF Tag:}

Radio Frequency identifying is done through the wireless and no-contact use of radio frequency electromagnetic fields to transfer data from transmitter to receiver or vice-versa, for identifying and tracking tags attached to objects. A Passive Clamp shell type tag is used for transmitting the road-sign which consists of memory chip and antenna.

\subsection{Speed Control Module:}

\section{a) 3.3.1 DC Motor:}

DC motor is a mechanically commutated electric device powered from direct current (DC). DC motors can operate directly from batteries which are rechargeable, providing them motive power for the first electric vehicles.

b) 3.3.2 Relay Module:

Electromechanical relays are the devices which join or break a circuit by physically moving the electrical contacts into contact with each other.

\section{c) RS232 to TTL converter}

The converter cables provide two way RS232 serial communication signal conversion between the TTL output (such as a micro controller board, like the Arduino) to and from a personal computer RS232 Serial COM port. So this converter may be used on any of the micro-controller which has TTL serial communications that must be converted to RS232.

\section{WORKING AND PROTOTYPE}

The main objective is to strap RFID tags to the road signs and affix sensors in the vehicle so that they can sense the tags, and provide information related to the tags to the driver and form Electronic Display Controller used for vehicle's speed control which is an embedded system. The RF tag can be fixed on an existing road sign to transmit the information provided by signals placed on the road to adapt the vehicle's speed. Once the information is received from the RF tags, the vehicle's Electronic Display Controller automatically warns the driver, to reduce the speed according to the traffic sign indicated by the tag. It waits for the driver's response to the information received, otherwise vehicle's EDC unit automatically reduces the speed. The Zone between two tags where the speed is controlled or managed is called as Sensitive Zone. This process can be used not only to indicate Sensitive zones but also provide some extra 
information to the drivers. Tags could give additional useful information such as locations of nearby hospitals, fuel stations and food centers, by serving as Navigator. If there is road work or a construction in progress in a locality, installment of a tag a few $\mathrm{km}$ before the distressed area can be used to suggest suitable detours, thereby averting potential traffic jams and blockades. The possibilities are numerous. Care should be taken to provide the alerts on a priority basis. The entire operation of the prototype is explained in the flow chart given below:

Flow chart for operation:

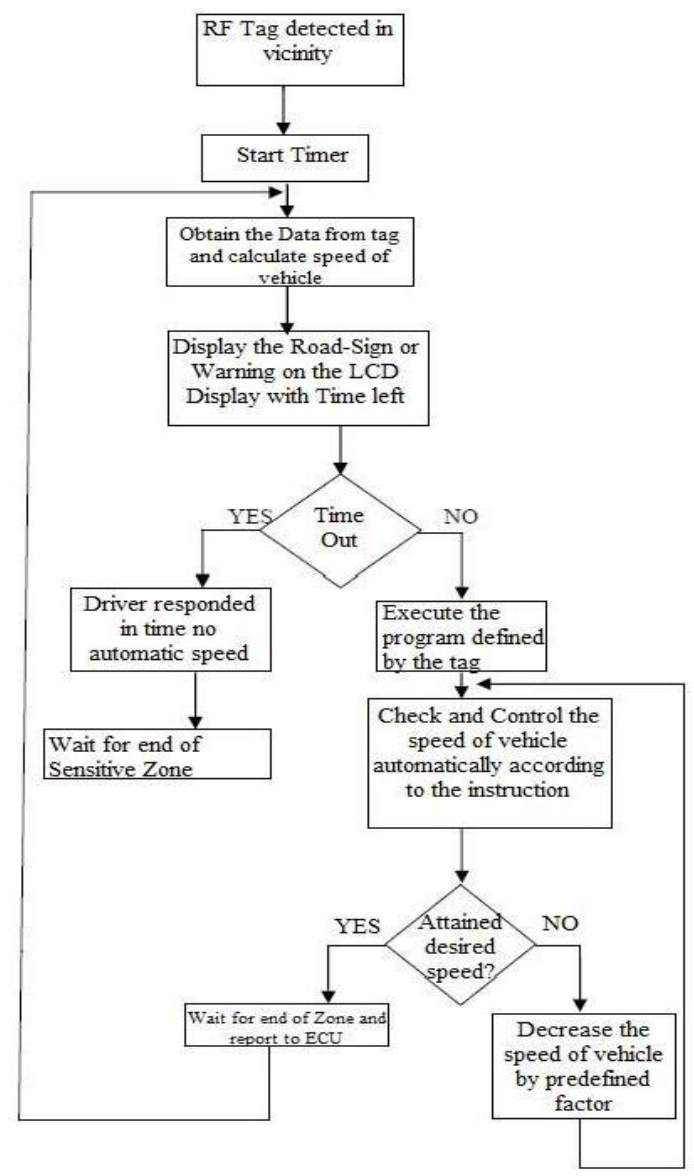

Figure 4.1: Flowchart

Different entities would require notification at different distances according to their proximities, which must be predetermined beforehand, to ensure that the driver is able to maneuver. Depending on the level of abnormality, the optimal distance must be fixed at the time of tag placement. So far it has explicitly and clearly explained the concept with one-way traffic only. In the real scenario, however, it is imperative to consider traffic bound in both oncoming and outgoing directions, with respect to the tag. This poses a potential problem. The tag must be readable from all directions. For example, a tag meant to be read by a vehicle traveling in the A-B direction may possibly be read by one traveling from B-A on the same road. Using of better directional Algorithms in programming of the micro controller and using of Active RF tags solves this problem with this prototype.

\section{EXPERIMENTAL ENVIRONMENT}

To validate the performance of the proposed method a model of vehicle is traversed at a speed higher than the speed written in the RFID transmitter, further arduino controlling the speed of vehicle. The Received Signal Strength Indicator (RSSI) helps to find out the strength of the signals received by the receiver. Thus it would be possible to check the strengths at different speeds. We can conclude from the strengths that higher the strength then higher are the signals received.

\section{IMPLEMENTATION}

The RFID tags are placed on the road parallel to the sign board When the car comes in the range of rfid tag, the threshold speed of the car is sent to the rfid reader which is further sent to the arduino. The arduino compares the speed of the vehicle with the speed sent as threshold. If the speed of the car exceeds threshold the the speed then the speed is reduced to the required speed in a controlled manner. An iterator is used to reduce the speed slowly and uniformly so as to avoid the jerky motion of the vehicle. The Developed system is shown in the Fig 6.1 where the electronic control unit is placed on the vehicle.

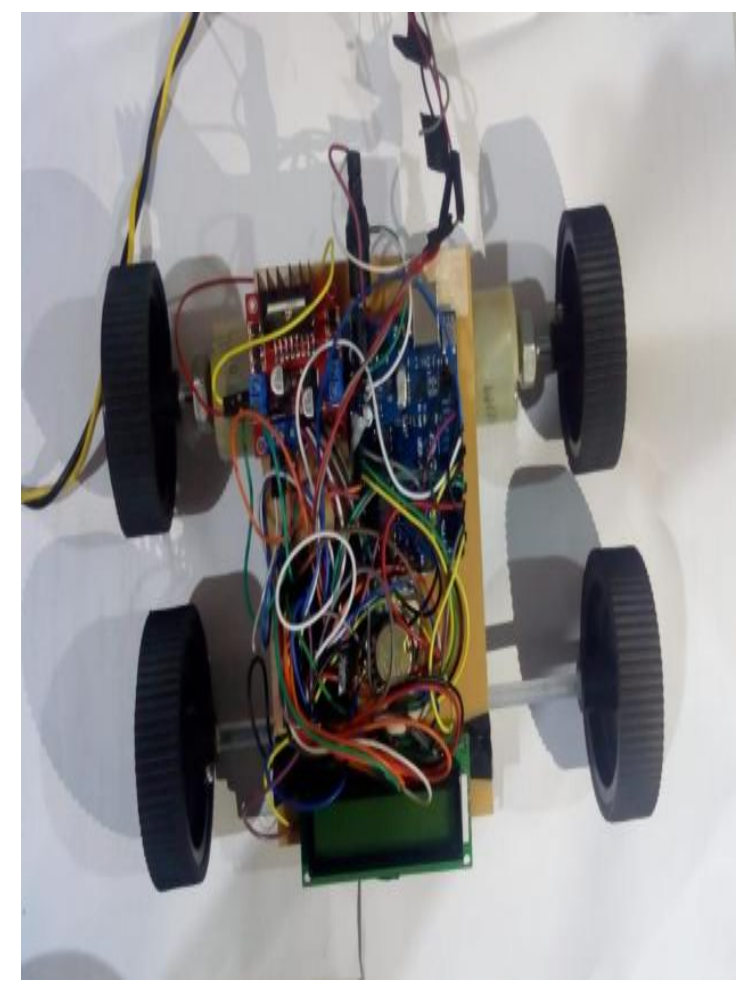

Figure 6.1 The developed model

\section{RESULTS AND DISCUSSION}

All the implementation of the existing as well as the proposed methods are done using RFID tags, arduino and EDC for displaying the results.

\section{CONCLUSION AND FUTURE WORK}

In this paper the prototype design of a system that can deliver road signs to drivers' vehicles and can control the speed of the automobile has been demonstrated. This project is very simple and low cost. This project consumes less power. This system is easy to implement on system present in current time which ensures more and more safety for drivers, passengers and pedestrians. In this way the driver can get the information that are sign boards without any kind of distraction or disturbance.. This prototype is perfect even for bad weather conditions lke smoggy, rainy, etc. While in contrast the technology of artificial 
vision-based recognition of traffic signals might fail if visibility is poor and GPS Navigation system may not work due to the distance issues of satellites or weak signals such as electromagnetic - Radio Frequency signals might still be transmitted reliably and efficiently through all the conditions. We are trying to work with the all boons and banes related to this project. In this prototype only one vehicle is considered. Practically other vehicles moving nearby can block or attenuate $\mathrm{RF}$ signals which may cause problem. This project may be enhanced further by establishing in-vehicle-vehicle microcontroller's communication through Radio-frequency waves so as to avoid vehicle collisions and to prevent deaths caused due to misleading signals. Some additional features like sending messages regarding the vehicle to its owner can also be possible.

\section{REFERENCES}

[1] Automatic Vehicle Speed Control With WirelessIn-Vehicle Road Sign Delivery System Using ARM 7,1.Gummarekula
Sattibabu, 2. B.V.V.Satyanarayan, 3.VV Satyanarayana Kona

[2] A design model for Automatic vehicle speed Control, International Journal for Computer Applications, volume35, No.9, December 2011.

[3] Design and Prototype of an In-Vehicle Road Sign Delivery System using RFID, $201212^{\text {th }}$ International Conference on ITS Telecommunications.

[4] Design of RF based Speed control system for vehicles, International Journal of Advanced Research in Computer and Communication Engineering Vol. 1, Issue 8, October 2012.

[5] Vehicle Speed Control using R.F. Technology, 5704017 , June 2006, SRM institue of Science and Technology. 